\title{
Suppression of 14-3-3 $\zeta$ in cholangiocarcinoma cells inhibits proliferation through attenuated Akt activity, enhancing chemosensitivity to gemcitabine
}

\author{
YINGPINYAPAT KITTIRAT ${ }^{1-3}$, ANCHALEE TECHASEN ${ }^{2,4}$, SUYANEE THONGCHOT $^{1,2}$, WATCHARIN LOILOME ${ }^{1-3}$, \\ RAYNOO THANAN $^{1,2}$, PUANGRAT YONGVANIT ${ }^{1-3}$, SAKKARN SUNGKHAMANON ${ }^{2-5}$, ATTAPOL TITAPUN ${ }^{2,3,6}$, \\ NARONG KHUNTIKEO ${ }^{2,3,6}$ and NISANA NAMWAT ${ }^{1-3}$ \\ ${ }^{1}$ Department of Biochemistry; ${ }^{2}$ Cholangiocarcinoma Research Institute; ${ }^{3}$ Cholangiocarcinoma Screening and Care Program; \\ ${ }^{4}$ Faculty of Associated Medical Sciences; Departments of ${ }^{5}$ Pathology and ${ }^{6}$ Surgery, Faculty of Medicine, \\ Khon Kaen University, Khon Kaen, Muang 40002, Thailand
}

Received November 5, 2016; Accepted September 28, 2017

DOI: $10.3892 / \mathrm{ol} .2017 .7326$

\begin{abstract}
The protein 14-3-3 $\zeta$ contributes important regulatory functions in several cellular processes via binding to phosphorylated serine/threonine residues, which promotes cell cycle progression, cell proliferation and anti-apoptosis in multiple types of cancer. The aim of the present study was to investigate the functions of $14-3-3 \xi$ in cholangiocarcinoma (CCA) progression and elucidate the molecular mechanism of 14-3-3 $\zeta$ expression-mediated protein kinase B (Akt) phosphorylation and chemosensitivity in CCA cells. In the present study, 14-3-3 $\zeta$ expression was investigated in clinical specimens using immunohistochemistry and compared with the clinicopathological features of patients with CCA. The association between 14-3-3 $\zeta$ and phosphorylated Akt (pAkt) was determined among the tissues of the same patients using bivariate correlation analysis. The effects of 14-3-3 $\zeta$ suppression on CCA cell function and gemcitabine sensitivity were investigated using small interfering RNA (siRNA). It was identified that 14-3-3 $\zeta$ expression was positively correlated with pAkt $(\mathrm{P}=0.013)$ and that increased expression of $14-3-3 \xi$ and pAkt were significantly associated with poor overall survival rate and metastasis $(\mathrm{P}=0.025$ and 0.006 , respectively). Downregulation of 14-3-3\} using siRNA in CCA cell lines decreased cell proliferation, resulting in the inhibition of pAkt activity and increasing the protein level of the cell cycle inhibitor $\mathrm{p} 27$. The suppression of 14-3-3 6 enhanced the inhibitory effect of gemcitabine on CCA cell proliferation by inducing apoptotic cell death. Taken together, the results of
\end{abstract}

Correspondence to: Dr Nisana Namwat, Department of Biochemistry, Faculty of Medicine, Khon Kaen University, 123 Mittraphap Road, Khon Kaen, Muang 40002, Thailand

E-mail: nisana@kku.ac.th

Key words: 14-3-3६, phosphorylated protein kinase B, p27, cholangiocarcinoma, chemosensitivity, gemcitabine the present study indicated that $14-3-3 \xi$ is a potential target for CCA and may serve as a novel therapeutic approach to enhance chemosensitivity in the treatment of CCA.

\section{Introduction}

Cholangiocarcinoma (CCA) is a cancer of the lining of the bile duct epithelium, the prevalence of which is increasing worldwide (1-3).CCA is a major public health problem in the northeast of Thailand where its etiology is strongly associated with liver fluke (Opisthorchis viverrini) infection that causes persistent bile duct inflammation (4). The incidence rates of CCA are between 93 and 318 per 100,000 individuals annually (5) with males being more commonly affected than females, and with an estimated 20,000 mortalities annually (6). CCA progression is relatively slow and patients frequently present at the hospital with late-stage disease in which the cancer has spread to other organs. Notably, chemotherapy in combination with surgery, rather than surgery alone, decreases tumor size and prolongs the patients' survival time (7). Therefore, the mechanisms, particularly alterations in molecular pathways that drive tumor cell functions during CCA progression, require further investigation in order to contribute to the improvement of guidelines for the treatment of CCA.

The protein $14-3-3 \zeta$ belongs to the $14-3-3$ protein family that exhibits an oncogenic potential through its interaction with target proteins involved in cancer initiation and progression (8). 14-3-3 $\zeta$ has been identified in several types of cancer, including breast (9), oral (10) and gastric (11) cancer, esophageal squamous carcinomas (12) and intrahepatic CCA (13), leading to apoptosis resistance, cancer recurrence and chemoresistance $(9,14)$. In contrast, 14-3-3 $\zeta$ small interfering RNA (siRNA) treatment of cancer cell lines sensitized cells to stress-induced apoptosis and effectively decreased the onset and growth of tumor xenografts (9). In addition, 14-3-3 knockdown by siRNA in lung cancer cells increased sensitivity to cisplatin in vitro and in vivo (15).

The molecular mechanisms by which $14-3-3 \zeta$ exerts its functions in cancer cells have been elucidated. Neal et al (16) 
reported that 14-3-3 $\zeta$ overexpression enhanced protein kinase B (Akt) phosphorylation via binding to the p $85 \alpha$ regulatory subunit of phosphoinositide 3-kinase (PI3K), an upstream signaling protein of Akt in breast cancer cell lines, contributing to cancer cell proliferation and survival. The PI3K/Akt signaling pathway is prominently activated in CCA, regulating tumor growth and metastasis, and its inhibitors may be potentially used as a targeted drug for the treatment of CCA (17). However, to the best of our knowledge, neither the functions of 14-3-3 5 regulating the PI3K/Akt signaling pathway nor its chemosensitivity in CCA cells have been reported previously.

In the present study, it was demonstrated that the co-expression of $14-3-3 \zeta$ and pAkt is associated with a poor prognosis of patients with CCA. Specific targeting of 14-3-3 $\zeta$ inhibited CCA cell proliferation via the suppression of pAkt activity and enhanced the chemotherapeutic effect of gemcitabine.

\section{Materials and methods}

Human CCA tissues. Tissue specimens from 75 patients with CCA admitted to Srinagarind Hospital, Khon Kaen University (Khon Kaen, Thailand) were collected. Of the 75 patients, $50(67 \%)$ were male and $25(33 \%)$ were female. The median age of patients was 57 years (range, 32-73 years) (Table I). The paraffin-embedded CCA tissues were obtained from the specimen bank of the Liver Fluke and Cholangiocarcinoma Research Center, Faculty of Medicine, Khon Kaen University, between January 1999 and December 2007. The protocol for collection and the study design were approved by the Ethics Committee for Human Research, Khon Kaen University (HE571283) and written informed consent was obtained from each subject prior to surgery.

CCA cell lines. The human intrahepatic CCA cell lines KKU-M213 and its derivative KKU-M214 were isolated from Thai patients with CCA and established in the Liver Fluke and Cholangiocarcinoma Research Center. Cells were cultured in Gibco ${ }^{\circledR}$ Ham's F-12 nutrient mixture (Gibco; Thermo Fisher Scientific, Inc., Waltham, MA, USA) supplemented with $10 \%$ Gibco ${ }^{\circledR}$ heat-inactivated fetal bovine serum (Gibco; Thermo Fisher Scientific, Inc.), $100 \mathrm{U} / \mathrm{ml}$ penicillin and $100 \mathrm{mg} / \mathrm{ml}$ streptomycin at $37^{\circ} \mathrm{C}$ in a humidified incubator containing $5 \% \mathrm{CO}_{2}$.

Immunohistochemical analysis. The expression of 14-3-3ל and pAkt was examined in CCA tissue sections using immunohistochemical (IHC) staining. The $4-\mu \mathrm{m}$-thick tissue sections were deparaffinized and rehydrated in an aqueous ethanol solution series. Antigen retrieval was performed by submerging slides in $10 \mathrm{mM}$ citrate buffer ( $\mathrm{pH}$ 6.0) and heating in a microwave oven at $100^{\circ} \mathrm{C}$ for $10 \mathrm{~min}$. The sections were blocked with $0.3 \%$ hydrogen peroxide followed by $10 \%$ skimmed milk at room temperature for $30 \mathrm{~min}$ for each blocking step. Subsequently, the sections were incubated with rabbit antibody against 14-3-3 (dilution, 1:1,000; cat. no. ab51129; Abcam, Cambridge, UK) and pAkt (Ser ${ }^{473}$; dilution, 1:1,000; cat. no. SAB4300042, Sigma-Aldrich; Merck KGaA, Darmstadt, Germany) at $4^{\circ} \mathrm{C}$ overnight. Subsequently, sections were incubated with horseradish peroxidase-conjugated EnVision anti-rabbit secondary antibody at room temperature for $1 \mathrm{~h}$ (undiluted; cat. no. K4003; Dako; Agilent Technologies, Inc., Santa Clara, CA, USA). Antigen-antibody complexes were developed with 3,3'-diaminobenzidine tetrahydrochloride substrate kit (Vector Laboratories, Inc., Burlingame, CA, USA) for $10 \mathrm{~min}$, and were counterstained with hematoxylin for $2 \mathrm{~min}$. The intensity of staining was scored as follows: 0 , negative staining; 1 , weak staining; 2 , moderate staining; and 3 , strong staining. The proportion was scored based on the percentage of stained cells as follows: 0 , all with negative staining, $+1,<25 \%$ positive, $+2,25-50 \%$ positive; and +3 , $>50 \%$ positive. The final score was assigned by multiplying the intensity score by the proportion score. The median of the final score was selected as a threshold value to determine the high and low expression of $14-3-3 \xi$ and pAkt.

Transient siRNA transfection. RNA interference (RNAi) was performed using a small fragment duplex of RNA (siRNA) which is specific to 14-3-3 $\zeta$ mRNA (SMARTpool ON-TARGETplus siRNA, ID L-003332-00-0005) containing four specific sequences, 5'-AGAAAGGGAUUGUCGAUC A-3', 5'-GCAGAUGGCUCGAGAAUAC-3', 5'-GCCCGUAGG UCAUCUUGGA-3' and 5'-AAAGACAGCACGCUAAUA A-3', and control sequences, 5'-UGGUUUACAUGUCGA CUAA-3', 5'-UGGUUUACAUGUUGUGUGA-3', 5'-UGG UUUACAUGUUUUCUGA-3' and 5'-UGGUUUACAUGU UUUCCUA-3' (GE Healthcare Dharmacon, Inc., Lafayette, CO, USA). The CCA cells, seeded at a density of $6 \times 10^{4}$ cells, were transfected with $50 \mathrm{nM}$ si14-3-3 $\zeta$ using Lipofectamine RNAiMAX (Invitrogen; Thermo Fisher Scientific, Inc.) in 6-well plates for 24, 48 and $72 \mathrm{~h}$ post-transfection. The protein level was determined using western blotting to confirm transfection efficiency. Cellular functions were determined upon siRNA transfection to CCA cells.

Gemcitabine sensitivitytesting in CCAcells. At $12 \mathrm{~h}$ post-seeding, gemcitabine (Fresenius Kabi Oncology Ltd., Haryana, India) was added at different concentrations $(0,0.0001,0.001,0.01$, $0.1,1,10$ and $100 \mu \mathrm{M})$ to CCA cell cultures. The doses of gemcitabine were selected on the basis of the half-maximal inhibitory concentration $\left(\mathrm{IC}_{50}\right)$ of its treatment of CCA cells. A concentration of $1 \mu \mathrm{M}$, which provided a time-dependent inhibitory effect, was selected to treat si14-3-3 $\zeta$-transfected cells at $24 \mathrm{~h}$ post-transfection. To determine cell viability, cells with combined treatments were cultured for additional time periods (24 and $48 \mathrm{~h}$ ). Cell viability and apoptosis assays were performed at the aforementioned time points.

Western blot analysis. Transfected cells were harvested and then lysed with radioimmunoprecipitation assay cell lysis buffer (150 mM NaCl, 0.5 M Tris-HCl pH 7.4, 1\% Tween-20, $1 \%$ sodium deoxycholate, $0.1 \% \mathrm{SDS}$ ) for $10 \mathrm{~min}$ on ice. The cell lysates were centrifuged at $4^{\circ} \mathrm{C}$ at $14,000 \mathrm{x}$ g for $10 \mathrm{~min}$. Protein concentration was determined using a bicinchoninic acid protein assay kit (Pierce; Thermo Fisher Scientific, Inc.). Protein extracts were solubilized in 4X SDS buffer containing dithiothreitol and were boiled at $95^{\circ} \mathrm{C}$. Protein was loaded $(20 \mu \mathrm{g} /$ well $)$ and separated on $10 \%$ polyacrylamide gel by SDS-PAGE prior to being transferred onto polyvinylidene fluoride membranes. 
Table I. Association between the co-expression of 14-3-3 $\zeta$ and pAkt $\left(\mathrm{Ser}^{473}\right)$ with clinicopathological characteristics of patients with CCA demonstrated by immunohistochemical staining.

\begin{tabular}{|c|c|c|c|c|c|}
\hline \multirow[b]{2}{*}{ Factor } & \multirow[b]{2}{*}{ No. of patients $(n=75)$} & \multicolumn{3}{|c|}{$\begin{array}{l}\text { Co-expression of } 14-3-3 \zeta \\
\text { and pAkt }\left(\mathrm{Ser}^{473}\right)\end{array}$} & \multirow[b]{2}{*}{ P-value } \\
\hline & & High & Low & Others & \\
\hline Age, years & & & & & 0.527 \\
\hline$<57$ & 36 & 18 & 5 & 13 & \\
\hline$\geq 57$ & 39 & 15 & 5 & 19 & \\
\hline Sex & & & & & 0.611 \\
\hline Female & 25 & 13 & 3 & 9 & \\
\hline Male & 50 & 20 & 7 & 23 & \\
\hline Histological type & & & & & 0.118 \\
\hline Papillary & 13 & 4 & 4 & 5 & \\
\hline Non-papillary & 62 & 29 & 6 & 27 & \\
\hline Overall metastasis & & & & & $0.006^{\mathrm{a}}$ \\
\hline Non-metastasis & 28 & 8 & 8 & 12 & \\
\hline Metastasis & 47 & 25 & 2 & 20 & \\
\hline
\end{tabular}

${ }^{\text {a }}<0.05$ was considered to indicate a statistically significant difference. pAkt, phosphorylated Akt.

The membranes were blocked with $5 \%$ skimmed milk at room temperature for $1 \mathrm{~h}$ and probed with each primary antibody at $4^{\circ} \mathrm{C}$ overnight, including rabbit anti-human 14-3-3 $\zeta$ (dilution, 1:1,000; cat. no. ab51129; Abcam), rabbit anti-human pAkt $\left(\mathrm{Ser}^{473}\right)$ (dilution, 1:1,000; cat. no. SAB4300042; Sigma-Aldrich; Merck KGaA), mouse anti-human PI3K p $85 \alpha$ (dilution, 1:2,000; cat. no. ab86714; Abcam), rabbit anti-human Akt (dilution, 1:1,000; cat. no. 9272; Cell Signaling Technology, Inc., Danvers, MA, USA), mouse anti-human p27 (dilution, 1:1,000; cat. no. 3698S; Cell Signaling Technology, Inc.). $\beta$-actin was used as a loading control (mouse anti-human $\beta$-actin; dilution, 1:10,000; cat. no. A5441; Sigma-Aldrich; Merck KGaA). Following incubation with horseradish peroxidase-conjugated secondary antibodies, including goat anti-rabbit (dilution, 1:2,000; cat. no. G21234) and rabbit anti-mouse (dilution, 1:4,000; cat. no. A16166; both Invitrogen; Thermo Fisher Scientific, Inc.) at room temperature for $1 \mathrm{~h}$, the band intensity was measured using enhanced chemiluminescence Prime Western Blotting Detection reagent (GE Healthcare, Chicago, IL, USA). The apparent density of the bands on membranes was captured by ImageQuant ${ }^{\mathrm{TM}}$ Imager (GE Healthcare).

Cell viability and apoptosis assays. The sulforhodamine B (SRB) assay (cat. no. S1402; Sigma-Aldrich; Merck KGaA) was used for determining cell viability in transfected KKU-M213 and KKU-M214 cells. Briefly, cells were seeded at a density of $2 \times 10^{3}$ cells in 96 -well flat-bottom microtiter plates and incubated for 24, 48 and $72 \mathrm{~h}$. Cells were then fixed with $10 \%$ trichloroacetic acid and stained with $0.4 \%$ SRB in $1 \%$ acetic acid for $45 \mathrm{~min}$. The protein-bound stain was solubilized with $10 \mathrm{mM}$ Tris base $(\mathrm{pH} 10.5)$ and absorbance was measured at $540 \mathrm{~nm}$ using a microplate reader (Sunrise, Tecan Group, Ltd., Mannedorf, Switzerland). The apoptotic cells were detected using an Annexin V-FITC and propidium iodide staining kit (Roche Diagnostics, Basel, Switzerland), according to the manufacturer's protocol. Stained cells were enumerated using FACSCanto II flow cytometry and analyzed using BD FACSDiva ${ }^{\mathrm{TM}}$ software, version 6.1.3 (BD Biosciences, San Jose, CA, USA).

Statistical analysis. Statistical analysis was performed using SPSS software (version 17.0; SPSS Inc., Chicago, IL, USA). A survival curve was calculated using the Kaplan-Meier estimator method. The association between 14-3-3 $\zeta$ and pAkt $\left(\mathrm{Ser}^{473}\right)$ and patients' clinicopathological data was analyzed using Fisher's exact test. The correlation between 14-3-3 with pAkt $\left(\operatorname{Ser}^{473}\right)$ was performed using a Spearman-rank correlation test. Statistical comparisons between two different groups were performed using unpaired Student's t-tests. The significance of the data compared between siRNA-treated and control groups was analyzed using one-way analysis of variance, followed by Fisher's least significant difference test. $\mathrm{P}<0.05$ was considered to indicate a statistically significant difference.

\section{Results}

Co-expression of 14-3-3\} and pAkt is associated with poor prognosis of patients with CCA. Of the 75 patients with intrahepatic CCA from whom CCA tissue was obtained, 50 $(67 \%)$ were male and $25(33 \%)$ female. The age of patients ranged from 32 to 73 years (median age, 57 years) (Table I). The histological types were classified as papillary in 13 (17\%) and non-papillary in $62(83 \%)$ cases. For all 75 CCA cases, positive immunohistochemical staining for $14-3-3 \zeta$ and pAkt was observed in the cytoplasmic region of the tissue sections (Fig. 1A-D). High co-expression of 14-3-3 $\zeta$ and pAkt was observed in 33 (44\%) cases, whereas low co-expression 

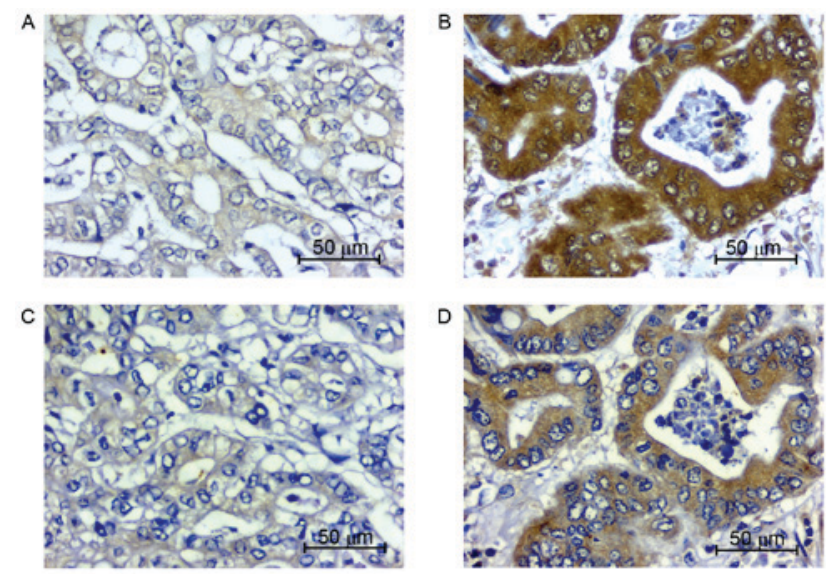

$\mathrm{E}$

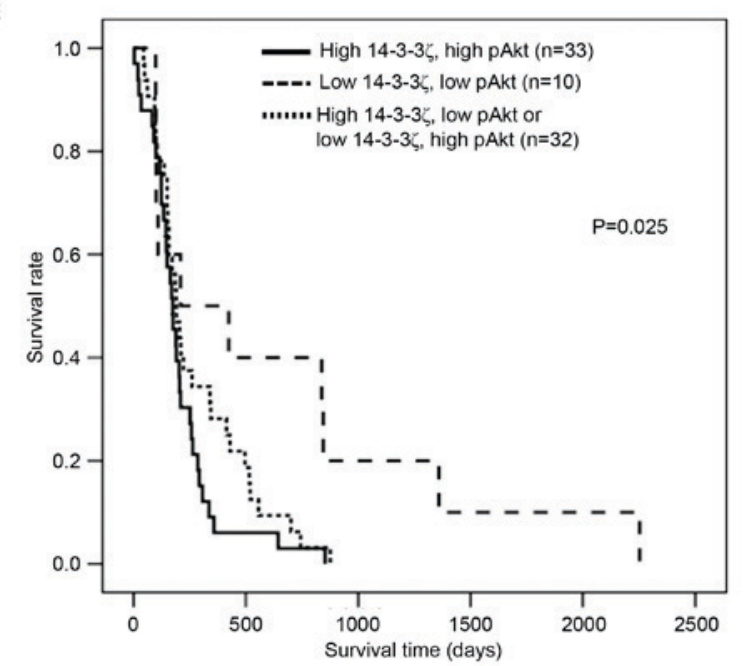

Figure 1. Expression of $14-3-3 \zeta$ and pAkt $\left(\mathrm{Ser}^{473}\right)$ in human CCA tissues illustrated by immunohistochemical staining. Staining in tumor cells: (A) low 14-3-3 $\zeta$ expression, (B) high 14-3-3 expression, (C) low pAkt (Ser ${ }^{473}$ ) expression and (D) high pAkt $\left(\mathrm{Ser}^{473}\right)$ expression. Magnification, $\mathrm{x} 400$. (E) High co-expression of 14-3-3 $\zeta$ and pAkt $\left(\mathrm{Ser}^{473}\right)$ indicate poor overall survival of patients with CCA as determined by the Kaplan-Meier estimator method Akt, protein kinase B; pAkt, phosphorylated Akt; CCA, cholangiocarcinoma; PI3K, phosphoinositide 3-kinase.

of 14-3-3 $\zeta$ and pAkt was observed in 10 (13\%) cases. High expression of 14-3-3z with low expression of pAkt was observed in 18 (24\%) and low expression of 14-3-3 $\zeta$ with high expression of pAkt was observed in 14 (19\%) cases. The immunohistochemical scores for $14-3-3 \xi$ and pAkt were analyzed for correlation using bivariate analysis. The results revealed that $14-3-3 \xi$ expression was positively correlated with pAkt expression $(r=0.287 ; \mathrm{P}=0.013)$.

There was a high co-expression of 14-3-3ל and pAkt associated with overall metastasis (Table $\mathrm{I} ; \mathrm{P}=0.006$ ). Age, sex and histological type were significantly different between these two groups. The cumulative survival rate, analyzed using the Kaplan-Meier estimator method, revealed that patients with CCA with high co-expression of $14-3-3 \zeta$ and pAkt $(n=33)$ had a significantly shorter survival time compared with those with low co-expression $(n=10)$ or inverse expression of $14-3-3 \zeta$ and pAkt ( $n=33$; $\mathrm{P}=0.025$; Fig. 1E).

Knockdown of 14-3-3\} attenuates the PI3K/Akt pathway and increases the p27 protein level. The 14-3-3 $\zeta$ siRNA (si14-3-3\}) and the negative control (siControl) were transfected into KKU-M213 and KKU-M214 cell lines. The successful suppression of 14-3-3 expression at $72 \mathrm{~h}$ was verified by western blot analysis, which identified suppression of 91\% in KKU-M213 (Fig. 2A and B) and 76\% in KKU-M214 (Fig. 2C and D) cells. To test the hypothesis that knockdown of 14-3-3 $\zeta$ affects the PI3K/Akt signaling pathway resulting in interference of the CCA cell cycle, the levels of pAkt $\left(\mathrm{Ser}^{473}\right)$, a downstream signaling molecule of PI3K, and the cyclin-dependent inhibitor p27, were assessed by western blotting in KKU-M213 (Fig. 2A and B) and KKU-M214 (Fig. 2C and D) cells. The results identified that the ratio of pAkt $\left(\operatorname{Ser}^{473}\right)$ to Akt was significantly decreased in si14-3-3 $\zeta$-transfected CCA cells when compared with siControl-transfected cells. si14-3-3 $\zeta$ transfection did not interfere with the PI3K p85 $\alpha$ level. Knockdown of 14-3-3 $\zeta$ inhibited cell cycle demonstrated by markedly increasing p27 protein levels was observed for transfected KKU-M213 and KKU-M214 cells at 48 and $72 \mathrm{~h}$ post-transfection.

Knockdown of 14-3-3ל leads to enhanced chemosensitivity to gemcitabine. It was investigated further whether knockdown of 14-3-3 ' may enhance the chemosensitivity of gemcitabine in CCA cells. KKU-M213 and KKU-M214 cells were transfected with si14-3-3 $\zeta$ with or without gemcitabine for 48 and $72 \mathrm{~h}$ and subjected to cell viability (Fig. 3) and apoptosis assays (Fig. 4). si14-3-3ל (50 nM) transfection significantly inhibited KKU-M213 cell viability by 10 and $29 \%$ at 48 and $72 \mathrm{~h}$ post-transfection, respectively, when compared with control cells (Fig. 3A). si14-3-3 $\zeta$ transfection inhibited KKU-M214 cell viability by 28 and $35 \%$ at 48 and 72 h post-transfection, respectively, compared with control cells (Fig. 3B). Furthermore, treatment with $1 \mu \mathrm{M}$ gemcitabine combined with $50 \mathrm{nM}$ si14-3-3 $\zeta$ transfection was analyzed, to test whether this combination was able to enhance the inhibitory effect on viability in CCA cells. KKU-M213 cell viability was inhibited by 2 and $19 \%$ at 48 and $72 \mathrm{~h}$ post-transfection, respectively (Fig. 3A), and KKU-M214 by 18 and $17 \%$ at 48 and $72 \mathrm{~h}$ post-transfection, respectively, when compared with control cells (Fig. 3B). Gemcitabine $(1 \mu \mathrm{M})$ combined with si14-3-3\} (50 nM) suppressed KKU-M213 cell viability by 15 and $53 \%$ at 48 and $72 \mathrm{~h}$ post-transfection, respectively, when compared with the control cells (Fig. 3A). For KKU-M214 cells, gemcitabine

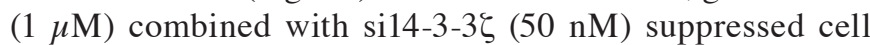
viability by 46 and $57 \%$ at 48 and 72 h post-transfection, respectively, when compared with the control cells (Fig. 3B). These results indicate that the combined effect of gemcitabine and si14-3-3 $\zeta$ was greater than the sum of the individual effects of treatments alone, compared with the control cells.

As presented in Fig. 4, KKU-M213 and KKU-M214 cells were transfected with si14-3-3 $\zeta(50 \mathrm{nM})$ for $24 \mathrm{~h}$ followed by gemcitabine treatment for an additional $48 \mathrm{~h}$ (i.e., $72 \mathrm{~h}$ post-transfection). Neither the si14-3-3 $\zeta$ (50 nM) transfection nor the gemcitabine treatment alone induced apoptosis in CCA cell lines at $72 \mathrm{~h}$ post-transfection when compared with the control cells. In contrast, gemcitabine $(1 \mu \mathrm{M})$ combined with si14-3-3 $\zeta(50 \mathrm{nM})$ significantly increased the number of apoptotic cells 2.8-fold in KKU-M213 (Fig. 4A and B) and 2.6-fold in KKU-M214 (Fig. 4C and D) when compared with the control cells. 

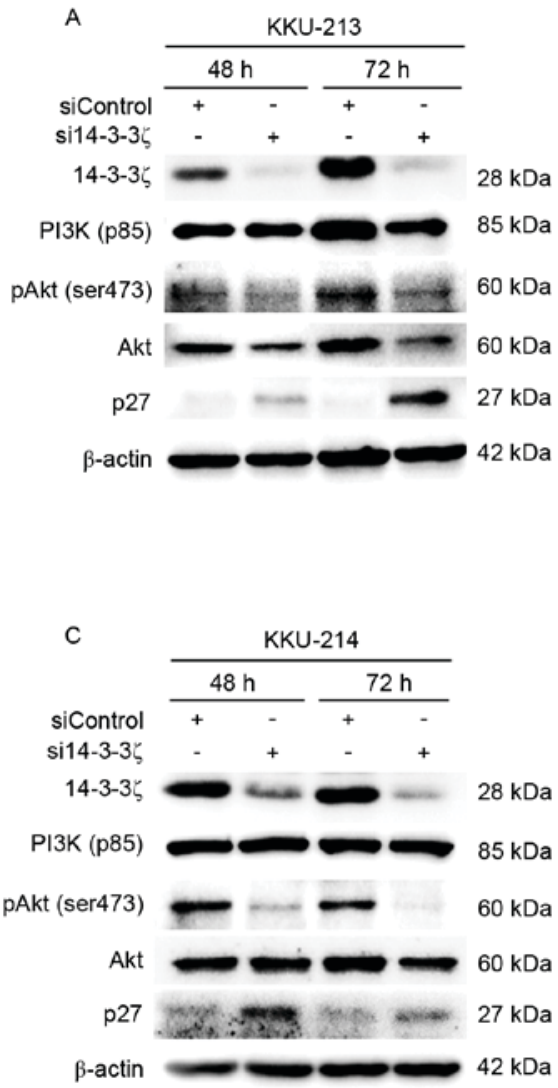

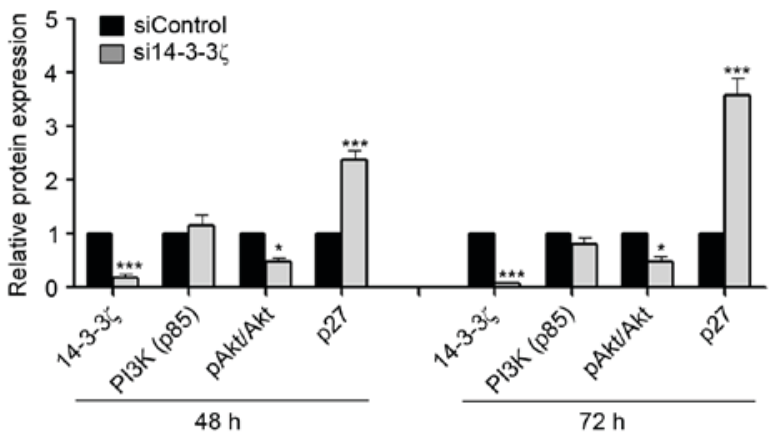

D

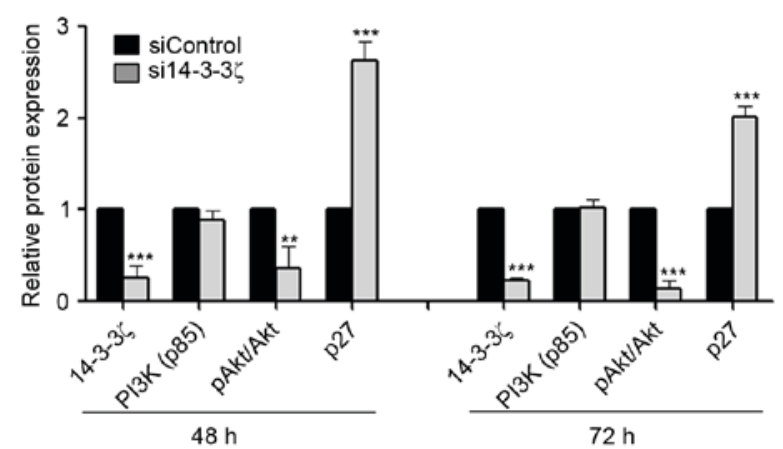

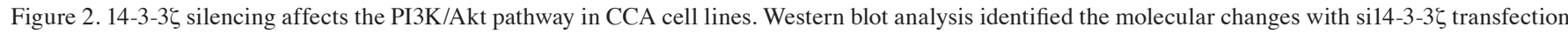
of (A) KKU-M213 cells at 48 and $72 \mathrm{~h}$ post-transfection. (B) Protein expression in KKU-M213 cells, relative to $\beta$-actin. Western blot analysis identified the molecular changes with si14-3-3̧ transfection of (C) KKU-M214 cells. (D) Protein expression in KKU-M214 cells, relative to $\beta$-actin. Akt, protein kinase B; pAkt, phosphorylated Akt; PI3K, phosphoinositide 3-kinase; CCA, cholangiocarcinoma; si, small interfering RNA. ${ }^{*} \mathrm{P}<0.05,{ }^{* *} \mathrm{P}<0.001$ and ${ }^{* * * *} \mathrm{P}<0.0001$, relative to $\beta$-actin.

A
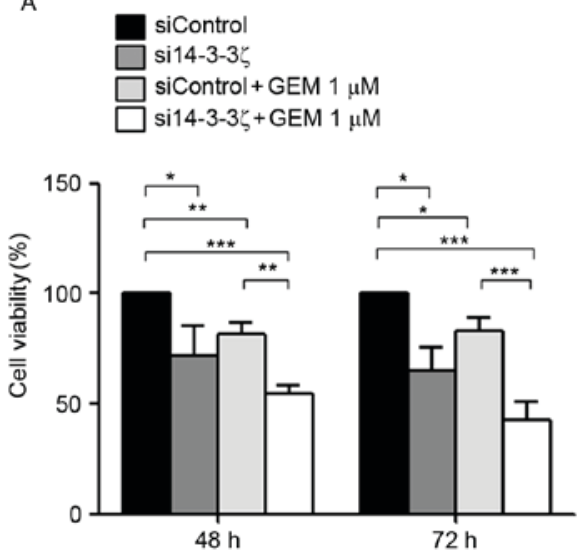

B
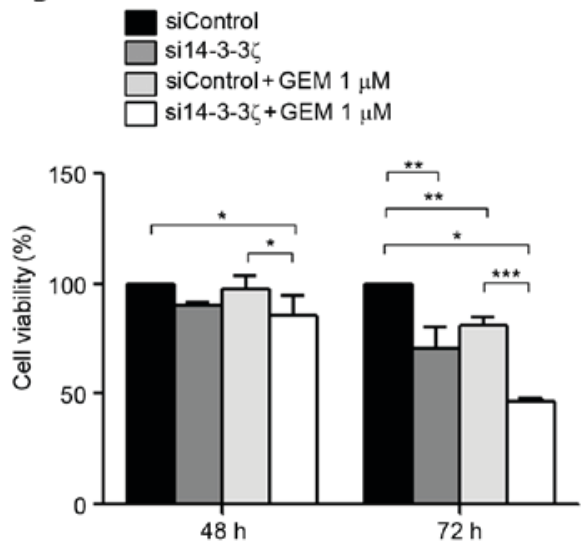

Figure 3. GEM decreases CCA cell viability. Suppression of 14-3-3६ enhances chemosensitivity to GEM. CCA cell lines were treated with $1 \mu$ M GEM at 48 and $72 \mathrm{~h}$ post-transfection. (A) KKU-M213 and (B) KKU-M214 were treated with $1 \mu \mathrm{M} \mathrm{GEM}$ at 48 and $72 \mathrm{~h}$ post-transfection and enhanced the chemosensitivity to GEM. Cell viability was determined using the sulforhodamine B assay. GEM, gemcitabine; CCA, cholangiocarcinoma; si, small interfering RNA. "P<0.05, ${ }^{* *} \mathrm{P}<0.001$ and ${ }^{* * * *} \mathrm{P}<0.0001$

\section{Discussion}

$14-3-3 \zeta$ acts as a regulatory protein that is able to interact with multiple client proteins that are involved in cancer initiation, progression and chemoresistance in multiple types of cancer (18). Several studies have demonstrated previously that overexpression of $14-3-3 \zeta$ in multiple types of cancer, including breast cancer (9), lung cancer (15) and intrahepatic CCA (13), is associated with poor prognosis of patients and may potentially serve as a novel molecular target for cancer treatment (19). The molecular mechanism by which 14-3-3 $\zeta$ serves a function in cancer cells has been 

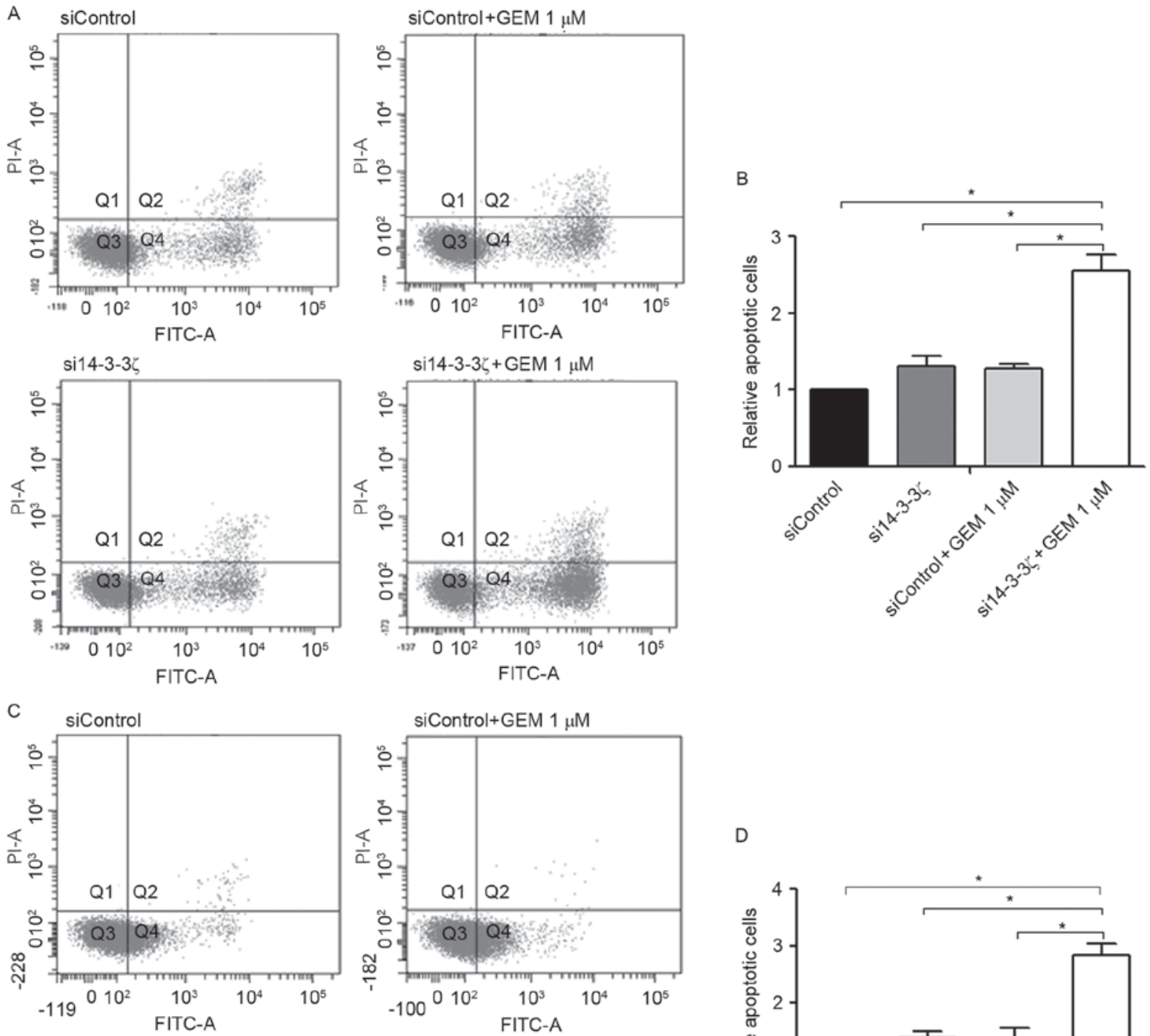

$\mathrm{D}$
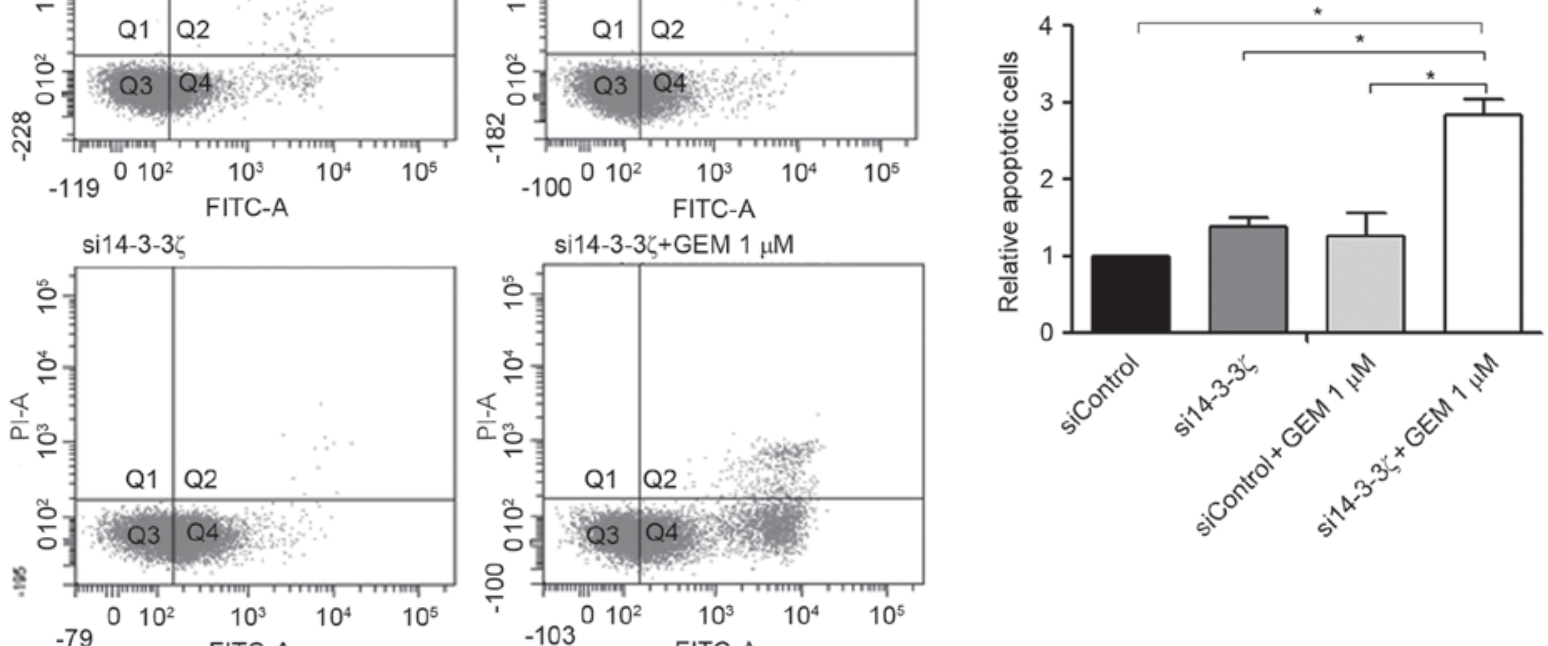

Figure 4. Suppression of 14-3-3ל enhances chemosensitivity to GEM by induction of apoptosis. (A) KKU-M213 cells were treated with $1 \mu \mathrm{M}$ GEM at $72 \mathrm{~h}$ post-transfection and apoptotic cells were enumerated by flow cytometric analysis using Annexin V and propidium iodide staining. (B) Relative apoptosis of KKU-M213 cells. (C) KKU-M214 cells were treated with $1 \mu \mathrm{M}$ GEM at $72 \mathrm{~h}$ post-transfection and apoptotic cells were enumerated by flow cytometric analysis using Annexin V and propidium iodide staining. (D) Relative apoptosis of KKU-M214 cells. FITC, fluorescein isothiocyanate; si, small interfering RNA; GEM, gemcitabine; si, small interfering RNA. "P<0.05.

investigated previously (16). It leads to an increase in the activation of the PI3K/Akt signaling pathway through binding with $\mathrm{Ser}^{83}$ on $\mathrm{p} 85 \alpha$. This leads to activation of the PI3K signaling cascade, contributing to proliferation and survival of human breast cancer cells (16). In addition, suppression of 14-3-3 $\zeta$ decreases the proliferative capacity caused by $\mathrm{S}$ phase arrest and promotes cell proliferation by activation of the extracellular-signal-regulated kinase signaling cascade and epithelial-mesenchymal transition induction in invasive QBC939 and RBE CCA cells (13). The results of the present study revealed that opisthorchiasis-associated CCA, in which the PI3K/Akt signaling pathway is predominantly activated, is linked with co-expression of 14-3-3 $\zeta$ and pAkt in CCA tissues. High scores for immunostaining of 14-3-3 $\zeta$ and pAkt were significantly correlated with a poor prognosis, suggesting that these molecules may serve as prognostic indicators for patients with CCA. The results of the present study are similar to those observed for breast cancer in which overexpression of 14-3-3 $\}$ was associated with increased Akt phosphorylation and correlated with a shorter survival time of patients (16). 
In the present study, the intracellular function of $14-3-3 \xi$ in CCA cells has been demonstrated. The suppression of 14-3-3 $\zeta$ attenuated CCA cell proliferation by inhibiting pAkt activity and inducing p27, leading to cell cycle arrest. However, suppression of $14-3-3 \xi$ to diminish pAkt activity was not sufficient to trigger apoptotic cell death. This is similar to a previous study by Yothaisong et al (17), in which inhibition of PI3 K/Akt using selective inhibitors was able to suppress CCA cell proliferation, but not induce apoptosis. Therefore, elimination of CCA cells by inhibiting this kinase signaling pathway requires multiple targeting strategies to gain therapeutic effectiveness.

CCA demonstrates an extreme resistance to therapeutic chemotherapies, targeted agents and radiotherapy. This intense resistance to a variety of therapies points to altered cell survival and metabolic pathways in these refractory types of cancer. Currently, gemcitabine appears to be an efficient therapeutic agent for patients with CCA $(7,20)$ as it exerts an inhibitory effect on CCA cell proliferation. Notably, it was revealed that 14-3-3 $\zeta$ knockdown synergistically enhanced gemcitabine sensitivity in CCA cells and induced substantial apoptotic cell death. The combined effect of gemcitabine and si14-3-3ל was greater than the sum of the individual effects on proliferative inhibition and apoptosis induction. This initial observation provides a new approach for CCA treatment by increasing the effectiveness of gemcitabine-based therapy. Further investigation using drug combinations between 14-3-3 inhibitors and gemcitabine in CCA cells is required in in vitro and in vivo studies.

In conclusion, high co-expression of $14-3-3 \zeta$ and pAkt was significantly associated with a poor prognosis for patients with CCA, indicating that combined $14-3-3 \zeta$ and pAkt may serve as prognostic indicators. Knockdown of 14-3-3\} inhibited pAkt $\left(\mathrm{Ser}^{473}\right)$ activity and increased $\mathrm{p} 27$, leading to suppression of CCA cell proliferation and induction of apoptosis. Combining siRNA treatment that targets 14-3-3 $\zeta$ with cytotoxic drugs, including gemcitabine may provide an effective strategy for the treatment of CCA.

\section{Acknowledgements}

The present study was supported by a Mid-Career Grant (grant no. RSA5980012), Thailand Research Fund, the Higher Education Research Promotion and National Research University Project of Thailand, Office of the Higher Education Commission, through the Health Cluster (SHeP-GMS) Khon Kaen University, a grant from Khon Kaen University, and a grant from the Faculty of Medicine, Khon Kaen University for supporting the M.Sc. program (grant. no. IN58218).

\section{References}

1. Khan SA, Emadossadaty S, Ladep NG, Thomas HC, Elliott P, Taylor-Robinson SD and Toledano MB: Rising trends in cholangiocarcinoma: Is the ICD classification system misleading us? J Hepatol 56: 848-854, 2012.

2. Bertuccio P, Bosetti C, Levi F, Decarli A, Negri E and La Vecchia C: A comparison of trends in mortality from primary liver cancer and intrahepatic cholangiocarcinoma in Europe. Ann Oncol 24: 1667-1674, 2013.
3. Ghouri YA, Mian I and Blechacz B: Cancer review: Cholangiocarcinoma. J Carcinog 14: 1, 2015.

4. Sripa B and Pairojkul C: Cholangiocarcinoma: Lessons from Thailand. Curr Opin Gastroenterol 24: 349-356, 2008.

5. Sriamporn S, Pisani P, Pipitgool V, Suwanrungruang K, Kamsa-ard S and Parkin DM: Prevalence of Opisthorchis viverrini infection and incidence of cholangiocarcinoma in Khon Kaen, Northeast Thailand. Trop Med Int Health 9: 588-594, 2004.

6. Bundhamcharoen K, Odton P, Phulkerd S and Tangcharoensathien V: Burden of disease in Thailand: Changes in health gap between 1999 and 2004. BMC Public Health 11: $53,2011$.

7. Valle JW, Wasan H, Johnson P, Jones E, Dixon L, Swindell R, Baka S, Maraveyas A, Corrie P, Falk S, et al: Gemcitabine alone or in combination with cisplatin in patients with advanced or metastatic cholangiocarcinomas or other biliary tract tumours: A multicentre randomised phase II study-The UK ABC-01 Study. Br J Cancer 101: 621-627, 2009.

8. Freeman AK and Morrison DK: 14-3-3 Proteins: Diverse functions in cell proliferation and cancer progression. Semin Cell Dev Biol 22: 681-687, 2011.

9. Neal CL, Yao J, Yang W, Zhou X, Nguyen NT, Lu J, Danes CG, Guo H, Lan KH, Ensor J, et al: 14-3-3zeta overexpression defines high risk for breast cancer recurrence and promotes cancer cell survival. Cancer Res 69: 3425-3432, 2009.

10. Matta A, Bahadur S, Duggal R, Gupta SD and Ralhan R: Over-expression of 14-3-3zeta is an early event in oral cancer. BMC Cancer 7: 169, 2007.

11. Nishimura Y, Komatsu S, Ichikawa D, Nagata H, Hirajima S, Takeshita H, Kawaguchi T, Arita T, Konishi H, Kashimoto K, et al: Overexpression of YWHAZ relates to tumor cell proliferation and malignant outcome of gastric carcinoma. Br J Cancer 108: 1324-1331, 2013

12. Bajpai U, Sharma R, Kausar T, Dattagupta S, Chattopadhayay TK and Ralhan R: Clinical significance of 14-3-3 zeta in human esophageal cancer. Int J Biol Markers 23: 231-237, 2008

13. Zhang C, Liu LX, Dong ZR, Shi GM, Cai JB, Zhang PF, Ke AW, Yu JX, Zhou J and Fan J: Up-regulation of 14-3-3zeta expression in intrahepatic cholangiocarcinoma and its clinical implications. Tumour Biol 36: 1781-1789, 2015.

14. Frasor J, Chang EC, Komm B, Lin CY, Vega VB, Liu ET, Miller LD, Smeds J, Bergh J and Katzenellenbogen BS: Gene expression preferentially regulated by tamoxifen in breast cancer cells and correlations with clinical outcome. Cancer Res 66: 7334-7340, 2006.

15. Fan T, Li R, Todd NW, Qiu Q, Fang HB, Wang H, Shen J, Zhao RY, Caraway NP, Katz RL, et al: Up-regulation of 14-3-3zeta in lung cancer and its implication as prognostic and therapeutic target. Cancer Res 67: 7901-7906, 2007.

16. Neal CL, Xu J, Li P, Mori S, Yang J, Neal NN, Zhou X, Wyszomierski SL and Yu D: Overexpression of 14-3-3zeta in cancer cells activates PI3K via binding the p85 regulatory subunit. Oncogene 31: 897-906, 2012.

17. Yothaisong S, Dokduang H, Techasen A, Namwat N, Yongvanit $\mathrm{P}$, Bhudhisawasdi V, Puapairoj A, Riggins GJ and Loilome W: Increased activation of PI3K/AKT signaling pathway is associated with cholangiocarcinoma metastasis and PI3K/mTOR inhibition presents a possible therapeutic strategy. Tumour Biol 34: 3637-3648, 2013.

18. Matta A, Siu KW and Ralhan R: 14-3-3zeta as novel molecular target for cancer therapy. Expert Opin Ther Targets 16: 515-523, 2012.

19. Matta A,DeSouzaLV,Shukla NK, GuptaSD, Ralhan R and Siu KW: Prognostic significance of head-and-neck cancer biomarkers previously discovered and identified using iTRAQ-labeling and multidimensional liquid chromatography-tandem mass spectrometry. J Proteome Res 7: 2078-2087, 2008.

20. Khan SA, Thomas HC, Davidson BR and Taylor-Robinson SD: Cholangiocarcinoma. Lancet 366: 1303-1314, 2005.

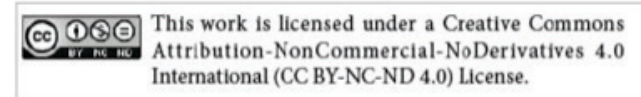

